\title{
Effect of Age, Gender and Vowel Type on Vowel Space Area in Sinhala Speakers
}

\author{
S.V. Narasimhan ${ }^{1 *}$ and W.G.S.S. Karunarathne ${ }^{2}$ \\ ${ }^{I}$ Department of Speech Language Pathology, JSS Institute of Speech and Hearing, \\ JSS Hospital, Karnataka, India \\ ${ }^{2}$ Wickramarachchi Speech and Hearing Institute, Nugegoda, Sri Lanka \\ Date Received: 18-02-2021_ Date Accepted: 16-03-2021
}

\begin{abstract}
This study was conducted to document the effect of age, gender and vowel type on vowel space area in Sinhala language. Three groups of participants were employed. Group 1 included 20 children, Group 2 included 20 adults and Group 3 consisted of 20 elderly subjects. All the subjects spoke the dialect of central province of Sri Lanka. Words consisting of three Sinhala short vowels /a/, /i/ and /u/ in were recorded. Formant frequencies of vowels were extracted and vowel space area was constructed. The results showed that the formant frequencies were significantly higher for children compared with those of adults. Female subjects had significantly higher formant frequency values than male subjects. Effect of vowel types were also significant on the formant frequencies and vowel space area. Sinhala also follows universal criteria of resonance characteristics and vocal tract constriction.
\end{abstract}

Keywords: vowel space area, formant frequencies, Sinhala, vowel articulation

\section{Introduction}

Vowel Space Area (VSA) is an acoustic measure that has been extensively used in quantifying vowel articulation. VSA is a graphical representation of vowel sounds and their position in both articulatory and acoustic space. The Euclidean distances between first formant frequency (F1) and second formant frequency $(\mathrm{F} 2)$ coordinates of the three corner vowels $/ \mathrm{a} /, / \mathrm{i} /$, and $/ \mathrm{u} / \mathrm{in}$ the $\mathrm{F} 1 / \mathrm{F} 2$ planes are considered for calculating VSA (Blomgren et al., 1998). Formants refer to the energy bands corresponding to the resonant properties of the vocal tract configuration (Chung et al., 2012).

The configuration, size, and shape of the vocal tract determine the formant frequencies of speech sounds. The back cavity of the mouth influences F1 and the front cavity of the mouth influences F2 (Peterson and Barney, 1952). Further, variation in F1 correlates with the height of the tongue while variation in F2 correlates with tongue advancement during vowel production (Hillenbrand, Getty, Clark, and Wheeler, 1995). Thus, limited range of F1 values for the high-low vowel contrast implies more restricted jaw and tongue vertical movements. Limited range of F2-F1 and low F2 values reflect reduced front-back movement of the tongue. Therefore, the 2-dimensional representation of vowel space area corresponds to tongue body position in an articulatory space. The hypothesis underlying the use of VSA is that greater vowel working space reflects better movement of the articulators with respect to tongue height (dimension of first formant frequency) or tongue advancement (dimension of second formant frequency) (Neel, 2008). VSA can thus be viewed as a powerful marker of vowel articulation and as an indicator of the accurateness with which a vowel is articulated. VSA being a quantitative acoustic index has several clinical applications.

*Correspondence: narasimhanslp@gmail.com

Tel: +919900789918

(C) University of Sri Jayewardenepura 
Extensive studies have been conducted on the various factors that affect VSA and formant frequency of vowels. These include age, gender and language. Peterson and Barney (1952) measured formant frequencies in both male and female subjects of various age ranges and reported highest formant frequencies in children followed by female subjects and lowest values of formant frequencies in male subjects. Eguchi and Hirish (1969) investigated formant frequencies across various age groups and concluded that there was a clear decrease in F1 and F2 in children from the age of 3 to 5 years. The decrease in F2 was higher compared to decrease in F1. The authors hypothesised that the development of the anterior part of the oral cavity probably had greater influence than the development of the posterior part of the oral cavity. They also opined that the formant frequencies were also influenced by psychophysiological factors.

Kent (1976) reviewed formant frequency data with reference to the neuromuscular and the anatomical and neuromuscular development of the speech mechanism and reported that the standard deviations of formant frequencies also progressively decreased with age in both these studies. Therefore, Kent (1976) concluded that the maturation of the motor skills in speech is not complete until the child reaches puberty. Vorperian and Kent (2007), performed a meta-analysis of formants and VSA in English speaking children and adults. They reported that the formants and VSA gradually reduced through childhood until adulthood. Flipsen and Lee (2012) also substantiated the gradual reduction of formants and VSA in children in their study.

Gender linked differences in vowel production have also been reported in the literature. Earlier studies have also reported significant effects of gender on the formant frequencies in children aged five years (Busby and Plant, 1995; Whiteside and Hodgson, 2000). A few researchers have documented significant gender linked differences in formant frequencies around 15 years of age (Eguchi and Hirish, 1969; Lee et al., 1999; Perry et al., 2001). Researchers have also reported larger vowel space area in females compared to males (Hillenbrand et al., 1995; Whiteside, 2001; Tsao et al., 2006; Flipsen and Lee, 2012). Studies suggest that these gender differences in vowels before the age of 10 years might be attributed to behavioural and social factors. Simpson (2009) suggested that the gender related differences in formant frequency and VSA in adult speakers may be due to the hyper articulated speaking style in women.

Apart from age and gender, formant frequencies and VSA of a specific vowel can also be affected by various factors such as size of a speaker, language characteristics, the rate of speech during vowel production, coarticulatory effects and phonation types (Peterson and Barney, 1952). Both intraspeaker and interspeaker variations can change the vowels to be phonemically different and thereby occupy various regions in the vowel space or may also cause the vowels to be phonemically identical and resulting in overlapping or occupying of indistinguishable regions in the vowel working space.

There have also been studies of F1, F2 and VSA in several languages. Most, Amir, and Tobin (2000) investigated the formant frequencies of Hebrew vowel system across age groups. Maximum difference in F1 was observed for the low vowel /a/ and minimal difference was observed for the high vowels /u/ and /i/ between the age groups reflecting that children had higher F1 values as compared to adults. Observations of F2 showed that the front vowels /e/ and /i/ had the maximal differences and back vowels /o/, /u/ had the minimal differences among groups. Thus, it was inferred that F2 was higher among children as compared to adults. Results also indicated higher F1 and F2 values for females as compared to males. The F2/F1 ratio also showed the similar results among groups. Man (2007) studied vowels in Hakka Chinese and reported lower F1 and F2 values for males compared to females. Krishna and Rajashekhar (2012) documented the variations of F1, F2 and VSA across the age among 72 Telugu speaking individuals in three different age groups. The results revealed higher F1, F2 and larger VSA in 
children compared to adults. The reduction in VSA as age advances was interpreted as being due to restricted vertical and horizontal movements of the tongue and the jaw.

Cross-linguistic studies on VSA and formant frequencies have revealed the differences in formant frequencies and vowel production across languages. Bradlow (1995) studied formant frequencies of vowels in adult speakers of English and Spanish and reported that the vowels systematically differed with respect to language. Vowels produced by English speakers had significantly higher F2 values compared to the vowels produced by Spanish speakers. Yang (1996) also investigated the vowels spoken by English and Korean adults and reported that English /a/ had lower F2 values than Korean /a/, and English /u/ had higher F2 values than Korean /u/.

Review of literature therefore shows that there have been extensive studies to demonstrate variations in VSA depending on age, gender and language. But much of the research comes from western languages. Even though VSA has possible clinical applications as a robust markers of vowel articulation and can be used as a quantifiable articulatory metrics ( Nikitha et al., 2015; Narasimhan et al., 2016; Narasimhan and Nataraja, 2019), there are no efforts to document VSA in Sinhala speaking population. Sinhala also called Sinhalese is an official language of Sri Lanka spoken by its majority population numbering roughly about 13 million people. It is an Indo-Aryan branch of the Indo-European language family. Thus, the present investigation aimed to document the effect of age, gender and vowel type on VSA in Sinhala speakers.

\section{Materials and Methods}

\subsection{Subjects}

The present study considered three groups of native Sinhala speaking subjects. Group 1 included 20 children in the age range of 7-11 years, Group 2 included 20 adults in the age range of 25-30 years and Group 3 consisted of 20 elderly subjects in the age range of 65-70 years. All the subjects spoke the dialect of central province of Sri Lanka. Each group consisted of ten male and ten female subjects. All the subjects were evaluated by an experienced Speech Language Pathologist to rule out any speech, language and cognitive disturbances. All the subjects had normal hearing sensitivity in the octave frequencies from 250 $\mathrm{Hz}$ to $8,000 \mathrm{~Hz}$ in both the ears (ANSI S3.21:2004, 2009) as per the audiometric testing. All the participants were monolingual native central province Sinhala speakers having no history of any hearing or language impairments. Informed consents were obtained from all the subjects or guardians/ parents in case of children.

\subsection{Test material}

The present study considered three Sinhala short vowels /a/, /i/ and /u/ as the target stimuli in initial, medial and final positions of the words. These three vowels were selected as they show good contrast in terms of high/low and front/back distinctions and also have clear differences in the F1/F2 space. A total of 27 picturable meaningful target words were chosen after doing familiarity check for 60 words with the lowest age group. Clusters and nasalised consonants were avoided during the selection of stimuli. The target vowels /a/, /i/, /u/ were embedded in either VCV/CVC/VCCV/CVCV context in the words. " $6 \times 4$ " dimension flashcards were prepared to elicit responses. Words were also written under the picture to elicit the exact response.

\subsection{Procedure}

Data recording was carried out in a classroom with low ambient noise at the end of the corridor in a school setup. A total of 27 target pictures were used in the study. A single picture was placed at a time on a table in front of the subject. Each subject was asked to name the picture when it was presented. The speech samples were recorded using Praat software version 5.3.23. (Paul Boersma and David Weenink; 
Institute of Phonetic Sciences, University of Amsterdam, Netherlands). All the speech samples were recorded using a dynamic, unidirectional microphone with frequency response of 60-12,000 Hz. The distance between the participant's mouth and the microphone was kept constant at a distance of $15 \mathrm{~cm}$. The recorded samples were digitised at a sampling frequency of $44.1 \mathrm{kHz}$ (16 bits/ sample quantisation).

\subsection{Data analysis}

The speech samples were analysed using Praat software. The vowels were identified and segmented via visual inspection of the spectrogram and by listening to the selected waveform. First formant frequency (F1) and second formant frequency (F2) were extracted from each vowel. Further, the averaged F1 and F2 values for each vowel were plotted on an F1/F2 plane to construct the vowel space area.

\section{Results}

The values of F1 and F2 were tabulated and statistically analysed using Statistical Package for the Social Sciences (SPSS 20; SPSS Inc., Chicago, IL, USA). As a part of descriptive statistics, mean and standard deviation values of F1 and F2 were obtained for all the subjects of all the groups. Further, Multivariate analysis of variance (MANOVA) was performed with 95\% confidence interval (using an alpha level of 0.05 ) to determine the effect of independent variables (Groups, Gender and Vowel) on the dependent variable (F1 and F2).

As it can be noted from Table 1 which shows the mean values of F1 and F2, the subjects of group 1 had higher mean F1 and F2 values compared to the subjects of groups 2 and group 3. Vowel /a/ showed highest F1 values and vowel /i/ showed lowest F1 values across all three groups. On the other hand, F2 values were observed to be higher for vowel /i/ compared to the other two vowels.

Table 1: Mean values of F1 and F2 across age groups and gender.

\begin{tabular}{lcrrrrrr}
\hline \multirow{2}{*}{ Variables } & \multirow{2}{*}{ Vowels } & \multicolumn{2}{c}{ Group 1 } & \multicolumn{2}{c}{ Group 2 } & \multicolumn{2}{c}{ Group 3 } \\
\cline { 2 - 7 } & & \multicolumn{1}{c}{ Male } & \multicolumn{1}{c}{ Female } & Male & Female & Male & Female \\
\hline \multirow{3}{*}{ F1 (in Hz) } & /a/ & 970.55 & 992.60 & 707.18 & 836.12 & 674.56 & 830.75 \\
& /i/ & 452.27 & 458.57 & 331.02 & 389.94 & 324.35 & 340.99 \\
& /u/ & 466.74 & 458.88 & 373.17 & 412.94 & 362.85 & 373.58 \\
F2 (in Hz) & /a/ & $1,700.36$ & $1,878.95$ & $1,352.57$ & $1,559.98$ & $1,274.37$ & $1,551.69$ \\
& /i/ & $2,952.95$ & $3,003.55$ & $2,246.25$ & $2,812.68$ & $2,246.86$ & $2,700.44$ \\
& $/ \mathrm{u} /$ & $1,142.63$ & $1,173.56$ & $1,008.17$ & $1,095.48$ & 948.85 & $1,014.02$ \\
\hline
\end{tabular}

Results of the MANOVA revealed that there was a significant effect of group on $\mathrm{F} 1(\mathrm{~F}=147.5$, $\mathrm{p}<0.05)$ and $\mathrm{F} 2(\mathrm{~F}=343.8, \mathrm{p}<0.05)$ values. Further, Scheffe post hoc test was performed to note the significant differences in F1 and F2 values between the three groups. It was evident from the results of the post hoc test that subjects of group 1 had the highest values of F1 and F2 compared to the subjects of both group 2 and group 3. It was interesting to note that there were no significant differences in F1 and F2 between the subjects of group 2 and group 3. Significant difference between genders across both the dependent variables was also noted. $\mathrm{F} 1(\mathrm{~F}=29.6, \mathrm{p}<0.05)$ and $\mathrm{F} 2(\mathrm{~F}=276.6, \mathrm{p}<0.05)$ values were significantly higher for female subjects than male subjects.

MANOVA results also revealed the significant differences between vowels for both $\mathrm{F} 1(\mathrm{~F}=1488.8$, $\mathrm{p}<0.05)$ and $\mathrm{F} 2(\mathrm{~F}=5677.6, \mathrm{p}<0.05)$ values. Scheffe post hoc test was carried out to find the significant differences in $\mathrm{F} 1$ between the three vowels /a/, /i/ and /u/. It was noted that vowel /a/ had significantly high $\mathrm{F} 1$ values compared to vowel /i/ and /u/. There was no significant difference in the F1 values between 
vowel /i/ and /u/. The results of the Scheffe post hoc test performed on the F2 values revealed that the vowel /i/ had significantly higher F2 values compared to other two vowels. However, there was no significant difference in the $\mathrm{F} 2$ values between vowel /a/ and /u/.

Further, vowel space area was constructed using the mean F1 and F2 values of vowels on an F1/F2 plane. F1 values were plotted on the $y$-axis and F2 values were plotted on the $\mathrm{X}$-axis. Figure 1 and Figure 2 display the vowel space area across the groups and gender respectively.

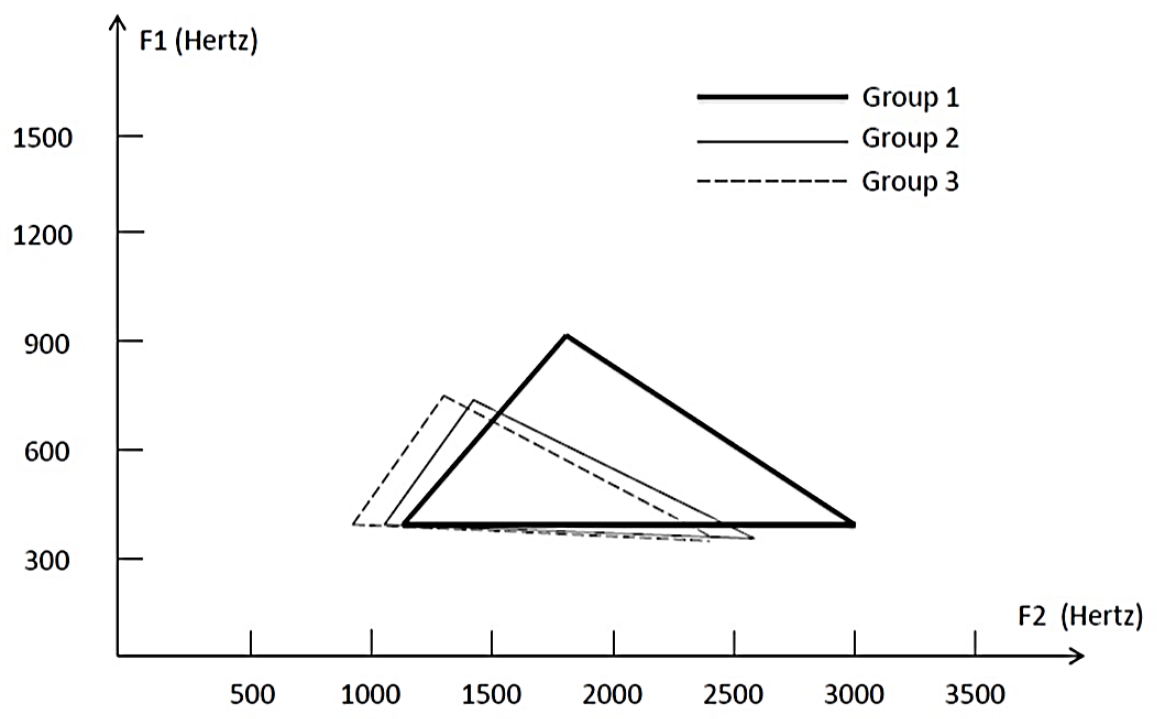

Figure 1. Vowel space area in subjects of all the three age groups.

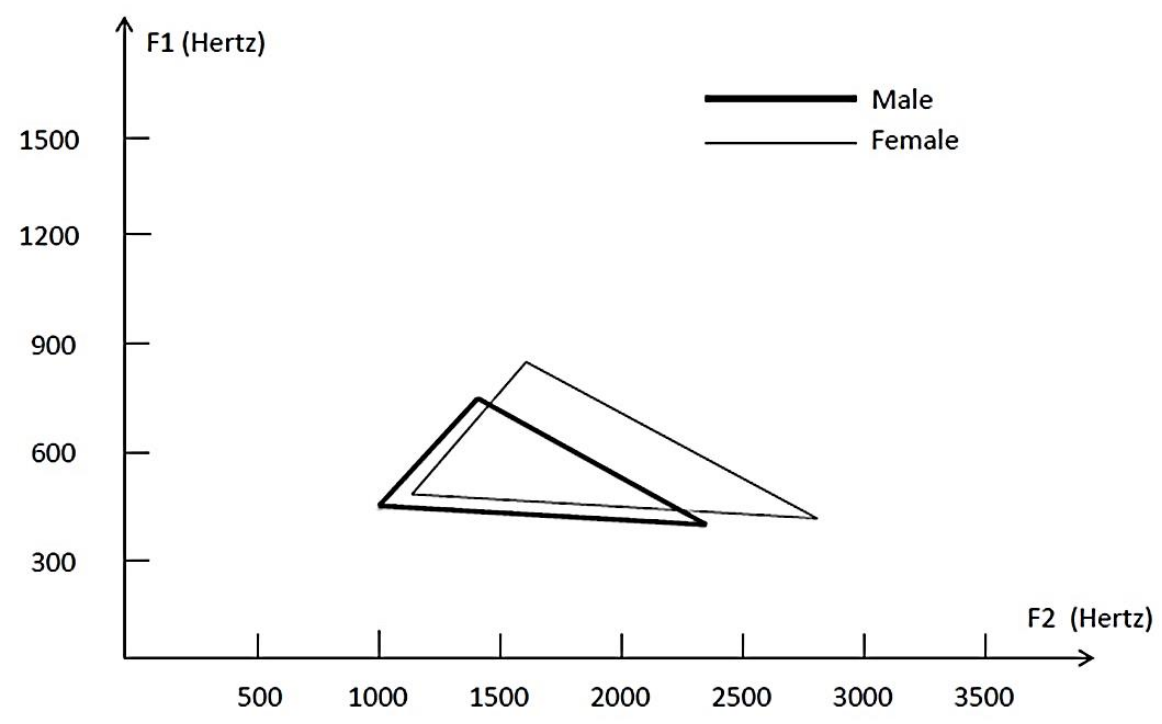

Figure 2. Vowel space area across gender.

As it can be noted from Figure 1, children had larger vowel space area compared to adult and elderly subjects. Among subjects of all the three groups, elderly subjects had the smallest vowel space area. From Figure 2, it was observed that the female subjects had larger vowel space area than male subjects. 


\section{Discussion}

Formant frequencies are related to tongue positioning and vocal tract constriction. According to Hillenbrand et al. (1995) and Peterson and Barney (1952), F1 variation principally correlated with tongue height in most of the languages. Similar results were observed in the present study. The central low mid vowel /a/ showed higher F1 values and front high vowel/i/ and back high vowel/u/ showed lower F1. Thus, the present study also confirms that the variation in F1 primarily correlates with tongue height during vowel production in Sinhala. Thus, the present study is completely in consonance with the earlier studies done by Hillenbrand et al. (1995) and Peterson and Barney (1952).

According to the literature review, F2 variation is primarily associated with tongue advancement during vowel production ( Peterson and Barney, 1952; Hillenbrand et al., 1995). Pickett (1996) also reported that F2 was lowered by the back tongue constriction and F2 was raised by the front tongue constriction. Therefore, given that F2 varies mostly with tongue advancement, anterior position of the tongue results in higher F2 and posterior tongue position results in lower F2 values. The analysis of F2 in the current study also reflected significantly higher values of F2 for the high front vowel /i/ compared to high back vowel /u/ and centre low vowel /a/. Thus, the results of the present study are in agreement with the earlier mentioned studies and also strongly support the findings that F2 variations are associated with the tongue advancement during vowel production in Sinhala.

Pettinato, Tuomainen, Granlund, and Hazan (2016) studied vowel space area in English speaking children for unscripted speech samples and reported that the vowel space area were larger in children compared to adults. Vorperian and Kent (2007) also reported gradual decrease in vowel formant frequencies and vowel space area as a function of age in English speaking children. The present study also evidenced that F1, F2 and VSA were larger in children as compared to adults and elderly subjects. Therefore it concurs with the concept that age-related changes documented in F1, F2 and VSA correlate with the age-related changes in the resonant property and the length of the vocal tract (Monsen and Engebretson, 1983).

Gender-related effects were also documented in the present study where females had a higher F1, F2 values and larger VSA compared to males. Therefore the results are in consonance with all the earlier studies documenting gender related differences in VSA ( Most et al., 2000; Whiteside, 2001; Flipsen and Lee, 2012; Krishna and Rajashekhar, 2012; Man, 2007; Tsao et al., 2006).

According to the acoustic theory (Fant, 1960), formant frequencies or optimal resonating frequencies reduce as the as the size of the vocal tract increases with age. Larger VSA in children may also be attributed to the articulatory characteristics in children. It can be hypothesised that larger articulatory movements in children may cause the vowels to occupy the extremes of the vowel space area (Green et al., 2002). Further, gender-related changes in F1, F2 and VSA could be attributed to the structural differences in the vocal tracts between genders.

The present study focused on picture naming task and did not consider spontaneous speech samples. Yet, from a normative point of view, the present investigation proves that the acoustic measures of formants and vowel space area are robust and reliable markers of vowel articulation as VSA data do not differ to a large extent between scripted and unscripted method of elicitation (Pettinato et al., 2016). Given that the dialectal variations can affect vowel characteristics, generalisations of the data ought to be made with some caution as the present study collected data only from speakers of central province dialect of Sinhala. Though limited, results of the present study can be used as a reference for other studies of speech and language disorders, speech processing strategies and speech synthesis among Sinhala speakers. Supplementary investigations on the normative aspects of formant frequencies and VSA among the 
Sinhala population would be provide additional insights and validated tools for indexing the vowel articulation.

\section{Conclusion}

The present study investigated the effects of age and gender on the formant frequencies and VSA among Sinhala speakers. Three Sinhala short vowels /a/, /i/ and /u/ were analysed for first and second formant frequencies and VSA were constructed on an F1/F2 plane. The results revealed that Sinhala follows universal criteria of resonance characteristics and vocal tract constriction. The results also revealed that the formant frequencies were higher for children compared with those of adults. VSA was also noted to be larger for children compared to adult and elderly speakers of Sinhala. Further, higher F1, F2 values and larger VSA was documented for female speakers as compared to males. Nevertheless, future studies could focus on investigating variation in formant frequencies and VSA as a function of age among Sinhala speaking children. The age and gender-related data of the Sinhala vowels acquired in the present investigation could assist as a preliminary reference in the assessment of communication impaired Sinhala speakers.

\section{References}

Blomgren, M., Robb, M.P., and Chen, Y., 1998. A note on vowel centralization in stuttering and nonstuttering individuals. Journal of Speech Language and Hearing Research Hearing Research, 41:1042-1051.

Bradlow, A.R., 1995. A comparative acoustic study of English and Spanish vowels. The Journal of the Acoustical Society of America, 97:1916-1924.

Busby, P.A., and Plant, G L., 1995. Formant frequency values of vowels produced by preadolescent boys and girls. The Journal of the Acoustical Society of America.

Chung, H., Kong, E.J., Edwards, J., Weismer, G., Fourakis, M., and Hwang, Y., 2012. Cross-linguistic studies of children's and adults' vowel spaces. The Journal of the Acoustical Society of America, 131:442-454.

Eguchi, S., and Hirish, I.J., 1969. Development of speech sounds in children. Acta Otolaryngologica Supplementum, 257:307-356.

Fant, G. 1960. Acoustic Theory of Speech Production. Mounton \& Co. N.V. Publishers, Fance.

Flipsen, P., and Lee, S., 2012. Reference data for the American English acoustic vowel space. Clinical Linguistics and Phonetics, 26:926-933.

Green, J.R., Moore, C.A., and Reilly, K.J., 2002. The sequential development of jaw and lip control for speech. Journal of Speech, Language, and Hearing Research, 45:66-79.

Hillenbrand, J., Getty, L.A., Clark, M.J., and Wheeler, K., 1995. Acoustic characteristics of American English vowels. The Journal of the Acoustical Society of America, 97:3099-3111.

Kent, R.D., 1976. Anatomical and neuromuscular maturation of the speech mechanism: Evident from acoustic studies. Journal of Speech and Hearing Research, 19:421-445.

Krishna, Y., and Rajashekhar, B., 2012. Vowel space area across age, gender, and dialects in Telugu. Languages in India.

Lee, P., and Narayanan., 1999. Acoustics of children's speech: Developmental changes of temporal and spectral parameters. The Journal of the Acoustical Society of America, 105:1455.

Man, C.Y., 2007. An acoustical analysis of the vowels, diphthongs and triphthongs in Hakka Chinese. International Congress of Phonetic Sciences.

Monsen, R.B. and Engebretson, A.M., 1983. The accuracy of formant frequency measurements: A comparison of spectrographic analysis and linear prediction. Journal of Speech and Hearing Research, 26:89-87. 
Most, T., Amir, O., and Tobin, Y., 2000. The Hebrew vowel system: raw and normalized acoustic data. Language and Speech, 43:295-308.

Narasimhan, S., and Nataraja, N., 2019. Vowel space area in speech of children with hearing impairment. International Journal of Health Sciences and Research, 9:97-102.

Narasimhan, S., Nikitha, M., and Nikita, F., 2016. Articulatory working space area in children with cerebral palsy. International Journal of Health Sciences and Research, 6:335-341.

Neel, A.T., 2008. Vowel space characteristics and vowel identification accuracy. J Speech Lang Hear Res, 51: 574-585. http://www.ncbi.nlm.nih.gov/entrez/query.fcgi?cmd=Retrieveanddb=PubMedanddo $\mathrm{pt}=$ Citationandlist_uids $=18506036$.

Nikitha, M., Nikita, F., and Narasimhan, S., 2015. Vowel space area in individuals with Parkinsons Disease. International Symposium Fontiers of Research on Speech and Music (FRSM).

Perry, T.L., Ohde, R.N., and Ashmead, D.H., 2001. The acoustic bases for gender identification from children's voices. The Journal of the Acoustical Society of America, 109:2988-2998.

Peterson, G.E., and Barney, H.L., 1952. Control methods used in a study of the vowels. Journal of the Acoustical Society of America, 24:175-184.

Pettinato, M., Tuomainen, O., Granlund, S., and Hazan, V., 2016. Vowel space area in later childhood and adolescence: Effects of age, sex and ease of communication. Journal of Phonetics, 54:1-14.

Pickett, J.M., 1996. The Sounds of Speech Communication- A Primer of Acoustic Phonetics and Speech Perception.

Tsao, Y.C., Weismer, G., and Iqbal, K., 2006. The effect of intertalker speech rate variation on acoustic vowel space. The Journal of the Acoustical Society of America.

Vorperian, H.K., and Kent, R.D., 2007. Vowel acoustic space development in children: a synthesis of acoustic and anatomic data. Journal of Speech Language and Hearing Research.

Whiteside, S.P., 2001. Sex-specific fundamental and formant frequency patterns in a cross-sectional study. The Journal of the Acoustical Society of America.

Whiteside, S.P., and Hodgson, C., 2000. Speech patterns of children and adults elicited via a picturenaming task: An acoustic study. Speech Communication.

Yang, B., 1996. A comparative study of American English and Korean vowels produced by male and female speakers. Journal of Phonetics. 